\title{
MODELO DE ANÁLISIS DE LA VULNERABILIDAD PSICOSOCIAL EN LA GESTIÓN DEL
} RIESGO DE DESASTRES

\author{
Katia Lucia Zapa-Pérez \\ Mestranda em Meio Ambiente e Desenvolvimento \\ Universidad Nacional de Colombia \\ Medellín - Antioquia - Colômbia \\ klzapap@unal.edu.co \\ Oscar Navarro Carrascal \\ Ph.D. em Psicologia \\ Universidad de Nantes \\ Nantes - França \\ oscar.navarro@univ-nantes.fr \\ Albeiro Rendón Rivera \\ Doctor en Ciencias de La Tierra \\ Universidad Nacional de Colombia \\ Medellín - Colômbia \\ arendonr@unal.edu.co
}

\section{RESUMEN}

En la problemática de los desastres, y más aún, en la reducción de la vulnerabilidad, existen vacíos en el conocimiento sobre los factores o variables psicosociales que juegan un rol importante en la forma como las personas identifican, evalúan e interpretan los riesgos. El objetivo de este artículo consiste en desarrollar un modelo de análisis de la vulnerabilidad ante el riesgo de desastres por fenómenos naturales o socio-naturales desde un enfoque psicosocial; para tal fin, se propone una definición de la vulnerabilidad psicosocial y se analiza la influencia de las variables psicosociales sobre la vulnerabilidad de una población en particular. El modelo se desarrolla a través de las variables: percepción del riesgo, representaciones sociales del riesgo, implicación personal, estrategias de afrontamiento (coping), apego al lugar y proximidad espacial. En este estudio se seleccionaron 257 participantes de sectores aledaños a la parte baja de la cuenca de la quebrada "El Barro", donde ocurrió una avenida torrencial en el 2005, y del sector de Niquía del Municipio de Bello Colombia. Los resultados muestran la influencia directa entre las variables psicosociales (correlaciones positivas y significativas); particularmente, una importante correlación entre la implicación personal y el coping activo. Esencialmente se concluye, primero, el modelo propuesto es pertinente desde el punto de vista conceptual y metodológico; segundo, la percepción a la acción frente a los riesgos incide significativamente en las estrategias de afrontamiento orientadas a la solución de problemas; terceiro, la experiencia previa de desastres influye de manera positiva sobre las variables psicosociales.

Palabras claves: Vulnerabilidad. Percepción del riesgo. Representación social del riesgo. Nivel de implicación personal. Estrategias de afrontamiento. Apego al lugar.

\section{MODEL OF ANALYSIS OF PSYCHOSOCIAL VULNERABILITY IN DISASTER RISK MANAGEMENT}

\section{ABSTRACT}

In the context of disaster problems and vulnerability reduction there exists some knowledge gaps about psycho-social factors or variables that play an important role in the way that people identify, evaluate and interpret disaster risks. The goal of this article is to develop a vulnerability analysis model for disaster risk by natural or socio-natural phenomena from a psychosocial approach. For this, a definition of psychosocial vulnerability is proposed and the influence of psychosocial variables in a particular population vulnerability are analyzed. The model is composed of six variables: risk perception, social representations of risk, personal involvement, coping strategies, place attachment and spatial proximity. In this study, 257 participants were selected from sectors bordering the lower part of the "El Barro" stream, where a torrential flood occurred in 2005, and from the Niquía sector of the Bello, Colombia. A direct influence between the psycho-social variables (positive and significant correlations) was found; in particular, there was an important correlation between personal involvement and active coping. Mainly conclusions are, first, the proposed model is conceptual and methodologically relevant; Second, action perceived about risks significantly affected coping strategies directed at solving the problems; third; previous experience with disasters influenced positively on psychosocial variables.

Key words: Vulnerability, risk perception, social representations of risk, personal involved, coping strategies, place attachment. 


\section{INTRODUCCIÓN}

Los desastres representan un gran reto para las sociedades contemporáneas. Durante las últimas décadas, los impactos generados por la intensidad de ocurrencia de fenómenos naturales han aumentado constante y considerablemente, principalmente aquellos asociados a eventos hidrometeorológicos (Cardona, Bertoni, Gibbs, Hermelin, \& Lavell, 2010). Estudios muestran un incremento significativo del riesgo de desastres derivado de las amenazas naturales y socionaturales entre 1990 y 2011 (ONU, 2013); en especial, en los países de bajos y medios ingresos que se sustentan en economías de rápido crecimiento (Yamin, Ghesquiere, Cardona, Ordaz, \& Mundial, 2013). Así mismo, se ha demostrado que el conocimiento insuficiente sobre las problemáticas de los riesgos constituye también uno de los elementos determinantes que inciden en la magnitud de los daños ocasionados por los desastres (Keipi, Mora, \& Bastidas, 2005). En consecuencia, desde hace décadas, el mundo científico y la comunidad internacional buscan entender y ampliar el conocimiento en relación al problema del riesgo de desastres, de manera que se pueda gestionar su reducción. Lo anterior se ha visto reflejado en la declaración de la Década Internacional de Reducción de Desastres Naturales (IDNDR) por parte de la ONU en 1990, al igual que en la Estrategia Internacional de Reducción de Desastres (ISDR) en el año 2000; y en las demás declaraciones derivadas de las conferencias mundiales para la reducción de Desastres celebradas en Yokohama, Hyogo y Sendai en los años 1994, 2005 y 2015 respectivamente.

Ahora bien, la ecuación del riesgo involucra la amenaza y la vulnerabilidad, por tanto modificar alguno de estos componentes, implica intervenir en el riesgo mismo (Cardona, 2001), sin embargo, las experiencias en desastres demuestran la necesidad de un análisis del riesgo más centrado en la vulnerabilidad que en la severidad del fenómeno amenazante; puesto que la prevención y mitigación del riesgo depende mayormente de las transformaciones que se dan en las condiciones de vulnerabilidad, que en la poca posibilidad que existe para intervenir en el fenómeno. Consecuentemente, un enfoque basado en la vulnerabilidad ha dado mayor claridad de los conceptos de riesgo y desastre (Cardona, 2001); así, este componente se convierte en un importante foco de análisis que contribuye al conocimiento y reducción del riesgo, y al fortalecimiento de las capacidades de una población, en cuanto a la preparación, respuesta y recuperación ante los impactos de los fenómenos peligrosos, que año tras año, impiden el desarrollo de la sociedades.

Actualmente se acepta que el riesgo de desastres es el resultado de las interacciones de la sociedad y su ambiente natural y/o artificial; por consiguiente, para profundizar en el riesgo se necesita entender tales interacciones y conocer los factores que las determinan (Cardona et al., 2010). En la vulnerabilidad de una comunidad también influyen factores intrínsecos al ser humano asociados a las configuraciones socio-cognitivas que se encuentran reguladas por las experiencias vividas, lo cognitivo, afectivo, social y cultural; y ésto, le da todo el sentido al análisis desde una perspectiva psicosocial. Consecuentemente, este artículo plantea la necesidad de incluir esta dimensión de análisis en el estudio del riesgo, donde el ser humano toma un lugar relevante, puesto que es quien proporciona el conocimiento sobre el pensamiento social e individual, los comportamientos y las interacciones dadas entre los miembros de una comunidad y su entorno; Así, este enfoque analiza los factores que intervienen en la forma cómo los individuos y grupos interpretan, evalúan y enfrentan el riesgo, teniendo como base el funcionamiento socio-cognitivo que opera en éstos, al igual que su interacción con el territorio que habitan.

Así, para efectos de la investigación se plantea una definición de la vulnerabilidad psicosocial, y conforme al objetivo que consiste en desarrollar un modelo de análisis de vulnerabilidad psicosocial en el contexto del riesgo de desastres, se construye y presenta un modelo conceptual que relaciona factores de la vulnerabilidad con dimensiones y variables psicosociales, el cual se pretende probar empíricamente en la población objeto de estudio. De este modo se parte de la hipótesis que sugiere la existencia de un tipo de vulnerabilidad directamente influenciada por variables que pertenecen a la dimensión psicosocial; y estas variables, se encuentran reguladas por las condiciones particulares de los individuos y grupos; como es el caso, de la exposición o no a una 
amenaza, y las experiencias previas o no de desastres; las cuales se usan en este estudio como criterio diferenciador de cada uno de los grupos seleccionados.

En este artículo se presentan seis secciones distribuidas de la siguiente manera: el capítulo 2 aborda el riesgo y la vulnerabilidad desde una perspectiva teórica psicosocial, y se propone una definición de la vulnerabilidad psicosocial como punto de partida para el análisis. En el capítulo 3 se presenta la metodología y el desarrollo conceptual del modelo de análisis de la vulnerabilidad psicosocial. Asimismo, el análisis estadístico y los resultados se muestran en el capítulo 4. Finalmente, la discusión y conclusiones se presentan en el capítulo 5 y 6 respectivamente.

\section{RIESGO Y VULNERABILIDAD: UNA PERSPECTIVA PSICOSOCIAL}

En las últimas décadas, las ciencias sociales y humanas, han desarrollado otras perspectivas de interpretación y evaluación del riesgo, disímiles a las sugeridas por las ciencias naturales y aplicadas. En efecto, ahora se reconoce que el riesgo objetivo se transforma a partir de la experiencia humana, lo cual hace que los individuos o grupos conciban el riesgo de acuerdo a sus imaginarios y percepciones (Narváez, Lavell, \& Pérez, 2009). En consecuencia, es de esperarse que el riesgo desde un punto de vista subjetivo, pocas veces coincida con las probabilidades reales de la peligrosidad y ocurrencia del fenómeno al que se teme, y con las estimaciones de la magnitud de su impacto.

En este sentido, se reconocen dos grandes enfoques del riesgo, marcado por lo objetivo y subjetivo, siendo este último muy disímil en sus características, al riesgo técnicamente evaluado u objetivo (Slovic, 1987). Así, la noción del riesgo se interpreta desde dos perspectivas: 1) la evaluación objetiva dada por la interacción entre los impactos dañinos de un peligro y la vulnerabilidad de las poblaciones frente a esos impactos; y 2) la evaluación subjetiva que involucra el análisis cognitivo que cada persona hace sobre el riesgo y que puede ser construida a lo largo de su vida (Lopez-Vazquez \& Marvan, 2012). No obstante, la evaluación subjetiva del riesgo también tiene un carácter colectivo; por tal razón, es también concebido como un fenómeno social que se compone por lo menos de tres dimensiones: la sociabilidad, la comunicación y el conocimiento social del riesgo colectivo (Gruev-Vintila \& Rouquette, 2007). Estudios realizados en distintas disciplinas como la antropología y la sociología han mostrado que existen aspectos sociales y culturales determinantes en la percepción y aceptación de los riesgos (Slovic, 1987).

Profundizar en el concepto del riesgo desde una dimensión psicosocial, significa entonces encontrar diferencias sustanciales en las formas empleadas por los diferentes actores de una sociedad para identificar y evaluar los riesgos. Teóricos del constructo psicométrico plantean que el ser humano usa esencialmente los sentimientos intuitivos e instintivos para evaluar los riesgos (Slovic \& Peters, 2006). Bajo este paradigma, estudios demuestran como dos emociones negativas: el miedo y la ira, producen respuestas opuestas en relación al riesgo; el primero amplifica el riesgo estimado y el segundo lo atenúa (Lerner, Gonzalez, Small, \& Fischhoff, 2003). Aunque este modelo aún es objeto de debate científico debido al cuestionamiento de sus bases empíricas (Sjöberg, 2002); esta línea teórica ha tenido aproximaciones científicas fundamentadas en métodos estadísticos, que responden a la atribución causal de los juicios, sentimientos, emociones y comportamientos de los individuos frente a los riesgos.

La vulnerabilidad por su parte, conceptualmente ha evolucionado en el tiempo. En la problemática de los desastres, la mayoría de las definiciones coinciden en que este concepto es una parte interna del riesgo y una característica intrínseca de los elementos en riesgo; sin embargo, este componente también tiene una connotación centrada en el ser humano, definida por la probabilidad de daños, muertes, lesiones, pérdidas y disrupción de los medios de vida ante los impactos negativos de un evento peligroso. Asimismo, se destaca una estructura dualística de la vulnerabilidad dada por la probabilidad de daños o afectación, la capacidad de afrontamiento, recuperación del estrés y de los impactos negativos del evento. De igual forma, este concepto se viste de una estructura múltiple, puesto que además de la susceptibilidad y capacidad de 
afrontamiento; se reconoce las capacidades adaptativas, la exposición y la interacción con la perturbación y el estrés. Y por último, la vulnerabilidad también se aborda desde una perspectiva multi-dimensional, en cuanto a que denota el cambio del análisis físico desde lo ingenieril para enfocarse en un análisis interdisciplinario y multidimensional que incluye características físicas, económicas, sociales, ambientales e institucionales (Birkmann, 2006).

La problemática del riesgo de desastres, se ha enfocado principalmente en el análisis dela vulnerabilidad física, social, económica, y últimamente, ambiental; dejando en evidencia un vacío en el conocimiento de la vulnerabilidad desde otras perspectivas cruciales para abordar este problema. Por consiguiente, este artículo además de proponer un modelo conceptual de análisis, ofrece a propósito una definición de vulnerabilidad psicosocial en el contexto del riesgo de desastres, desde el punto de vista de los autores. En efecto, se define la vulnerabilidad psicosocial como las características intrínsecas del funcionamiento socio-cognitivo de los individuos os grupos sociales que les permite identificar y evaluar los riegos; así mismo, esta organización sociocognitiva, regula la posibilidad de ser afectados ante la ocurrencia de un evento peligroso, y a su vez, determina la capacidad que ellos mismos reconocen tener para anticipar, preparar y recuperarse de las consecuencias de dicho evento. Así, la característica más sobresaliente de la vulnerabilidad psicosocial, es entonces, el orden interno y el carácter subjetivo de las evaluaciones que se suscitan a nivel individual y colectivo, y que son moduladas por lo cognitivo, afectivo, social y cultural.

\section{METODOLOGÍA}

El desarrollo metodológico de este estudio es cuantitativo con un enfoque exploratorioanalítico, no experimental. Los participantes se seleccionaron con base al grado de conocimiento y experiencia frente al objeto de estudio. Por tal razón, se usó una estrategia de muestreo intencional, la cual permite escoger a las personas que facilitan en mayor proporción entender el problema y las preguntas de investigación (Creswell, 2002). Todos los análisis parten de la información recolectada en campo en el segundo semestre del año 2015, mediante un cuestionario diseñado como el instrumento de medición de las variables estudiadas. Se aplicaron un total de 257 cuestionarios diligenciados en papel y el procesamiento estadístico se realizó mediante el software SPSS 16.0®.

Con el fin de verificar la pertinencia del modelo se realiza un análisis estadístico de los datos recolectados y se procede a realizar las respectivas pruebas estadísticas de validación del constructo y propiedades psicométricas del instrumento de medición; para lo cual se calcula la fiabilidad del instrumento (alfa de Cronbach) y su validez de constructo. Además, se analiza el grado de correlación existente entre las variables psicosociales del modelo, a partir de la matriz de correlación de Pearson, y se realiza la comparación múltiple de diferencia de las medias de los grupos por medio de la técnica ANOVA por un factor, para verificar el efecto de la experiencia y exposición como un factor explicativo.

\subsection{Construcción de modelo conceptual de la vulnerabilidad psicosocial}

Un enfoque psicosocial de la vulnerabilidad parte del supuesto que son las personas quienes tienen mayormente la capacidad de reducir los riesgos existente en su territorio; y de igual manera, prepararse, responder y sobreponerse ante las consecuencias fatales de los desastres; es decir, este enfoque concibe al ser humano como la base y el impulsor de los procesos que determinan y modifican sus condiciones de vulnerabilidad, y aumento de su resiliencia. Consecuentemente, se parte de una primera hipótesis que plantea la influencia de variables en el orden psicosocial sobre la vulnerabilidad de una comunidad; adicionalmente y acorde con la revisión de literatura que sugiere la repercusión que tiene la experiencia previa de desastres y la exposición ante una amenaza sobre las evaluaciones que las personas hacen frente al riesgo, se pretende probar una segunda hipótesis que consiste en el hecho que la experiencia previa de desastre por avenida torrencial en este caso, y la exposición ante la amenaza de este fenómeno, tiene un efecto modulador, ya sea positivo o 
negativo, sobre las variables psicosociales estudiadas, y por ende en la vulnerabilidad de una población.

En este orden de ideas, se desarrolla una propuesta de análisis que toma como base los tres factores determinantes de la vulnerabilidad, identificados por Cardona (2001): la exposición o condición de susceptibilidad del asentamiento humano (E), la fragilidad social (FS), y la falta de resiliencia o de capacidad de respuesta y recuperación (CR). A partir de estas consideraciones, los autores proponen un modelo conceptual de análisis que establece las relaciones existentes entre los factores de la vulnerabilidad y sus respectivas dimensiones y variables psicosociales (véase figura $1)$.

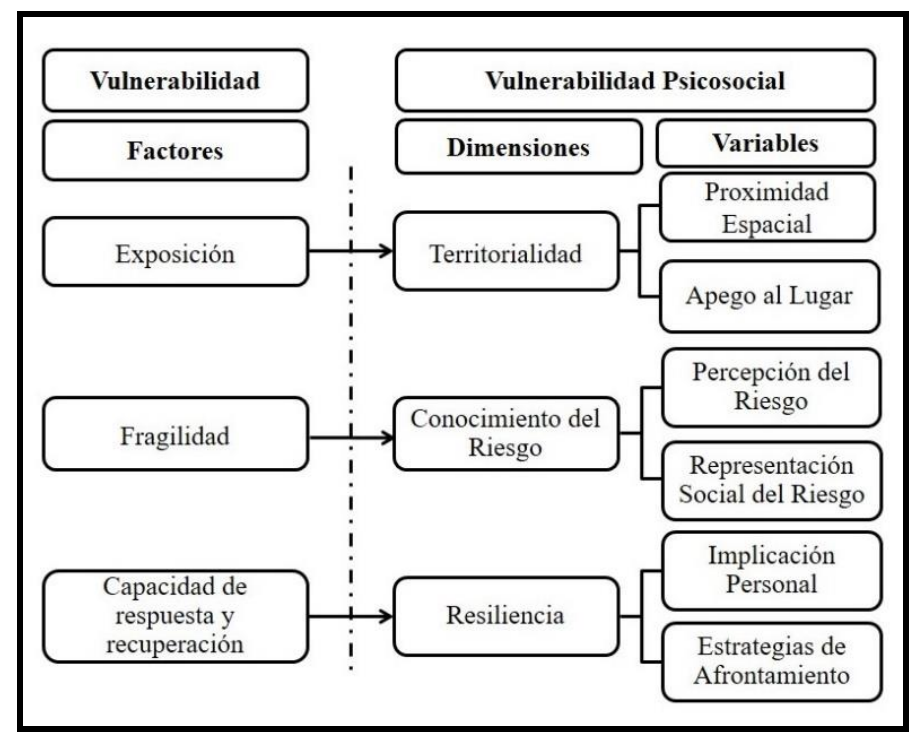

Figura 1. Modelo de la vulnerabilidad psicosocial. Fuente: Elaboración propia

El modelo propuesto se constituye en tres dimensiones, cuatro variables de carácter sociocognitiva y dos variables de tipo socio-territorial denominadas de la siguiente manera: 1) Dimensión territorialidad: engloba las variables, proximidad espacial y apego al lugar; 2) Dimensión conocimiento del riesgo: abarca las variables, percepción del riesgo y representaciones sociales del riesgo; y 3) Dimensión resiliencia: consta de las variables, nivel de implicación personal y estrategias de afrontamiento (coping). A continuación se exponen conceptualmente cada una de las dimensiones y sus respectivas variables.

\subsubsection{Territorialidad: Relación con el espacio de vida}

En el modelo se incluyen variables de tipo socio-territoriales dado que todo riesgo tiene una naturaleza territorial, en la medida en que se ubica espacialmente o geográficamente en el territorio; así se puede decir que muchos riesgos son propios de un territorio en particular, dependiendo de los fenómenos amenazantes que existan. Este espacio de vida condiciona a los seres humanos, en cuanto al desarrollo de su mundo cognitivo, afectivo, social y cultural, y a su vez, influye en sus condiciones de vulnerabilidad. Esta dimensión se constituye por las siguientes variables:

- $\quad$ Proximidad espacial: desde la perspectiva del riesgo, la distancia o proximidad a una amenaza ha sido objeto de estudio; investigaciones hacen referencia al efecto halo en cuanto a la subestimación del peligro que hacen la personas que viven en riesgo (Bickerstaff, 2004; CatalánVázquez, Riojas-Rodríguez, Jarillo-Soto, \& Delgadillo-Gutiérrez, 2009); en contraste otros estudios muestran que la cercanía a una amenaza conduce a un percepción más alta del riesgo (Lindell, Zhang, \& Hwang, 2009). Lo anterior sugiere que existe algún tipo de influencia de la escala geográfica sobre la evaluación del riesgo; y así lo demuestra un estudio realizado en México, donde las personas que vivían en las zonas más cercanas al cráter volcán Popocatépetl percibían el riesgo 
con mayor preocupación; sin embargo, el riesgo volcánico fue evaluado de manera más importante para las poblaciones que se encontraban más alejadas del volcán (Lopez-Vazquez, Marvan, Flores, $\&$ Peyrefitte, 2008). Esta variable entonces se refiere a la cercanía geográfica a un atributo que represente un fenómeno peligroso, y tiene una equivalencia a la exposición física. Cobra un interés especial a nivel psicosocial, ya que en el análisis de los riesgos no es posible extraer al ser humano de su territorio, por tanto es necesario considerar un escenario espacial para entender las relaciones entre los habitantes y su lugar de vida, al igual que las decisiones que éstos toman para elegir vivir en riesgo y expuestos a los impactos de eventos peligrosos. De igual modo, esta variable tendría una relación directa con el apego al lugar, debido a que las personas escogen estar próximos a los lugares con los que sienten apego (Hidalgo \& Hernández, 2001).

- Apego al lugar (APL): Distintas definiciones se encuentran asociadas a este concepto; Shumaker y Taylor (1983) definen esta noción como "lazos afectivos positivos o asociaciones entre los individuos y su ambiente residencial"; Hummon (1992) por su parte, hace referencia a "la implicación emocional con los lugares"; y Low (1992) lo asocia a la "conexión cognitiva y emocional hacia un lugar en particular" (citados por Hidalgo \& Hernández, 2001). Otros autores afirman que el apego al lugar se encuentra mediado por vínculos cognitivos y comportamentales establecidos por los individuos en relación a su ámbito físico y social (Brown \& Perkins, 1992), e incluso alguno teóricos adoptan un modelo cultural en el que sobresale el apego simbólico del individuo o grupo a determinados lugares (Giuliani \& Feldman, 1993). Asimismo, el apego al lugar abarca un conjunto de significados, identificación y afectos que ligan a las personas con sus entornos (Lewicka, 2011). Estudios han mostrado que el apego al lugar se constituye en un amplificador de la percepción de los riesgos de alta probabilidad, pero tiene un efecto contrario con los de baja probabilidad (Bernardo, 2013). Otras investigaciones muestran que existe una relación importante entre el apego al lugar y la voluntad de emprender acciones, lo cual puede depender del tipo de apego (Lewicka, 2011). En esta misma línea, una investigación en la India indica que el apego al lugar influye significativamente en la preparación frente a las inundaciones, y también muestra un efecto diferenciador dado por el tipo de apego (Mishra, Mazumdar, \& Suar, 2010). Con base a estudios se ha llegado a la conclusión que cuando hay un fuerte apego al lugar, cualquier ambiente, incluso a sabiendas que es muy peligroso, puede ser percibido como suficientemente bueno para vivir (Billig, 2006).

En síntesis, esta variable indaga sobre las evaluaciones que las personas hacen del lugar donde viven, y además, acerca de la resistencia que éstos tienen en abandonar los espacios que habitan, aunque implique vivir en riesgo. De esta manera, se constituye en un indicador explicativo de la permanencia de los pobladores, en el espacio y tiempo, en lugares específicos del territorio.

\subsubsection{Conocimiento del Riesgo}

Actualmente se acepta que el riesgo es socialmente construido, por consiguiente en su análisis, además de tener en cuenta las percepciones individuales, el funcionamiento sociocognitivo de los individuos, que bien se pueden expresar en los niveles altos o bajos de percepción, sentimiento de ilusión, invulnerabilidad o efecto halo de proximidad, es fundamental incluir estudios sobre el pensamiento social, los juicios, las evaluaciones que a nivel colectivo se hace en torno a los riesgos, ya que influyen significativamente en su conocimiento e interpretación, y además, orientan las acciones y comportamientos de las personas frente a los riesgos. Esta dimensión de análisis abarca las siguientes variables:

- Percepción del riesgo (PR): El paradigma psicométrico (Fischhoff, Slovic, Lichtenstein, Read, \& Combs, 1978) y la teoría cultural (Douglas \& Wildavsky, 1982), son enfoques bien conocidos y utilizados en la investigación sobre la percepción del riesgo. Investigadores de las ciencias sociales reconocen otros factores influyentes en las respuestas de las personas frente a las fuentes de peligros, tales como las creencias, actitudes, juicios, valores, disposiciones sociales y culturales (Puy \& Aragonés, 1997). Además de estas características 
individuales, algunos teóricos también incorporan las representaciones colectivas; por tanto, consideran que la percepción del riesgo es la representación de valores colectivos y el significado de acontecimientos, prácticas, elementos sociales y ambientales (López-Vasquez, 2001). En este mismo sentido, otros autores consideran que la percepción del riesgo también está influenciada por la plataforma cultural de las sociedades y por la forma como el riesgo se les comunica a las personas (Douglas \& Wildavsky, 1982; Slovic, Fischhoff, \& Lichtenstein, 1980).

El modelo psicométrico busca explicar cómo los individuos evalúan, temen o sienten, cuando se enfrentan a condiciones amenazantes o peligrosas; por tanto indaga fundamentalmente sobre los aspectos cognitivos y emocionales que tienen las personas frente a los riesgos (GruevVintila \& Rouquette, 2007); además, pretende ser una herramienta efectiva para predecir la percepción del riesgo, y ha tenido un avance importante en la organización de la estructura cognitiva individual y colectiva; actualmente es el más utilizado por investigadores (Kellens, Zaalberg, Neutens, Vanneuville, \& De Maeyer, 2011). En efecto, estudios realizados bajo este modelo han arrojado importantes resultados que muestran como el conocimiento de los individuos frente a los fenómenos amenazantes marcan las diferencias de los juicios que éstos emiten (Wandersman \& Hallman, 1993).

Este paradigma pone en relevancia una estructura cognitiva que subyace en los individuos independientemente del pensamiento y de la cultura; esta estructura la conforman básicamente dos factores: 1) el temor al riesgo, a lo desconocido, incontrolable, los impactos negativos, el riesgo para las generaciones venideras, la exposición involuntaria; y 2) el (des)conocimiento del riesgo que relacionado con lo que no se observa, lo novedoso y desconocido para las personas expuestas, al igual que para los expertos (Slovic et al., 1980). Estos autores sostienen que el temor al riesgo y el (des)conocimiento del mismo tienen un gran impacto en las evaluaciones que las personas hacen del riesgo, en sus comportamientos y en las decisiones que toman. En investigaciones sobre la percepción del riesgo por inundación, se encontró que los niveles de temor estuvieron especialmente influenciados por las emociones negativas y positivas de las personas en relación a sus anteriores experiencias de amenazas por inundación (Terpstra, 2009).

- $\quad$ Representaciones sociales del riesgo (RSR): la teoría de las representaciones sociales (RS) instaura el carácter social en el proceso de construcción del conocimiento, y este concepto se desarrolla en torno al pensamiento social y su organización cognitiva. La RS se define como "una modalidad particular del conocimiento, cuya función es la elaboración de los comportamientos y la comunicación entre los individuos" (Mora, 2002). Otros autores plantean que son "construcciones sociales de saberes ordinarios elaborados a partir de valores y creencias compartidos por un grupo social, dando lugar a una visión del mundo que se manifiesta en el seno de las interacciones sociales" (Navarro, 2004).

Un concepto más amplio marcado por el pensamiento social, sugiere la RS como el saber del sentido común que se constituye de un conocimiento específico y un pensamiento práctico que orienta la comunicación y las relaciones con el entorno social, material e ideal (Jodelet, 1986). La teoría de la RS se fundamenta en dos postulados principales: la existencia de una "sociedad pensante" y la existencia de una realidad "representada"(Abric, 1994). En otras palabras, toda realidad social está representada e incorporada en los sistemas de creencias, valores, juicios de los grupos y reestructurada en los procesos cognitivos de los individuos. Esta variable se incluye en el modelo, ya que se parte de la premisa de que toda realidad es representada y que existe una construcción social del conocimiento del riesgo.

Debido a que se usó un método específico para el análisis de esta variable, los resultados respectivos se presentarán en una publicación posterior.

\subsubsection{Resiliencia}

Esta dimensión es de gran importancia en la problemática de los riesgos, y para este propósito describe la capacidad que tienen los individuos y grupos en la preparación, respuesta y 
recuperación de los impactos de los desastres, y en adaptación ante las nuevas circunstancias de vida. Para el análisis de la resiliencia, se acude a indagar en los factores socio-cognitivos, puesto que éstos dan cuenta de las evaluaciones que las personas hacen de su propia capacidad de respuesta y adaptabilidad al riesgo. Las variables que incluyen esta dimensión se describen a continuación:

- Implicación personal (IP): a medida que una persona se encuentra más involucrada frente a un riesgo, el conocimiento de éste se enriquece. La implicación personal es un factor psicosocial que responde a lo subjetivo y social, en tanto que corresponde a la relación que establece el individuo con un objeto social; tiene una definición operacional basada en tres dimensiones que pueden medirse en una escala específica (Gruev-Vintila \& Rouquette, 2007); éstas son: 1) la valoración, evalúa la importancia del problema en los individuos;2) la identificación, hace referencia al grado de identificación de la persona en relación al objeto; 3) la percepción de posibilidad de acción (sentimiento de control), está ligada con la posibilidad de intervención de los individuos y con la evaluación de la capacidad de acción percibida por éstos hacia el problema.

Como se conoce teórica y empíricamente, las prácticas sociales juegan el papel más importante en las dinámicas del conocimiento del riesgo, y hay evidencias empíricas que comprueban que estas prácticas dependen o son reguladas por la implicación que tengan los sujetos frente al objeto social (Gruev-Vintila \& Rouquette, 2007). Consecuentemente, se puede afirmar que la implicación personal determina las prácticas sociales ligadas al riesgo; esto quiere decir que mientras menos implicados se encuentren los sujetos ante los riesgos, serán más escasas las acciones y decisiones que tomen para prevenir, reducir o mitigar los mismos. Esta variable se convierte en un buen indicador en el reconocimiento de la posibilidad de acción de los individuos expuestos a un peligro, y a su vez, aporta significativamente en las medidas de reducción del riesgo y en la recuperación del impacto de un desastre.

- $\quad$ Estrategias de afrontamiento (coping): La adaptación a los ambientes de amenazas son determinados por los recursos personales que les permiten a los individuos protegerse a sí mismos física y psicológicamente (Lopez-Vazquez, 2009); y ante un evento o en los momentos de crisis, el sujeto desarrolla estrategias para afrontar y manejar las condiciones de vida que les causa estrés y ajustarse a la nueva situación (Lazarus, 1999). De esta manera, el afrontamiento (coping), regula, estabiliza y mantiene la adaptación psicosocial de los individuos durante los periodos de crisis o estrés. Esta noción tiene su fundamento en las teorías del estrés; y se define como el conjunto de "esfuerzos cognitivos y comportamentales por los cuales el sujeto está destinado a gestionar las exigencias especificas internas o externas, que ponen a prueba o exceden los recursos de la persona" (Lazarus \& Folkman, 1986). Los teóricos del coping señalan que en la relación individuo-entorno, son tan importantes las características de la persona, como la naturaleza de los eventos que suceden en su ámbito de vida (Lazarus \& Folkman, 1984); sin embargo, la forma como los individuos perciben los eventos y problemas, es decir, el estrés percibido, en ocasiones es más relevante que el evento mismo; por esta razón, algunos autores plantean que la evaluación subjetiva de la situación es más importante que el mismo impacto (Lindsay \& Norman, 1980).

Existe una categoría del afrontamiento que surgen a partir de dos conceptos: el método usado y la focalización de respuesta; el primero usa respuestas activas o evitativas; y el último se orienta hacia la solución de problemas o control de emociones (Feuerstein, Labbé, \& Kuczmierczyk, 1986). En esta misma línea, se propone una tipología del afrontamiento basada en dos tipos: 1) afrontamiento activo, se orienta más a la solución de problemas, y está relacionado con comportamientos que enfrentan directamente la amenaza, tales como la recopilación de información, acciones directas, control o mitigación; 2) afrontamiento pasivo, se relaciona con la emoción, y corresponde a los comportamientos que implica rechazo, negación del evento, retiro o aceptación pasiva (Moos \& Billings, 1982; Lopez-Vazquez \& Marvan, 2012).

Estudios han destacado la influencia de la percepción del riesgo sobre las respuestas de estrés y de afrontamiento en una situación de riesgo catastrófico natural o industrial (LópezVázquez \& Marván, 2003); investigaciones similares, mostraron que un alto porcentaje de personas 
expuestas a los peligros volcánicos no se sentían preparadas para enfrentar un evento eruptivo y no tenían las estrategias para enfrentar los riesgos percibidos (Lopez-Vazquez, 2009).

\subsection{Localización del área de Estudio}

El estudio se realizó en el municipio de Bello, localizado en el norte del Valle de Aburrá, departamento de Antioquia, noroeste de Colombia; específicamente se seleccionó la zona piloto correspondiente a la cuenca de la quebrada "El Barro", debido a la ocurrencia del evento de avenida torrencial el 6 de octubre de 2005; este fenómeno es conocido por el común de las personas de la zona como avalancha. La ubicación del área de estudio se muestra en la figura 2, y los puntos de muestreo que corresponden a la parte baja de la cuenca y al sector Niquía del municipio, se pueden observar en la figura 3.

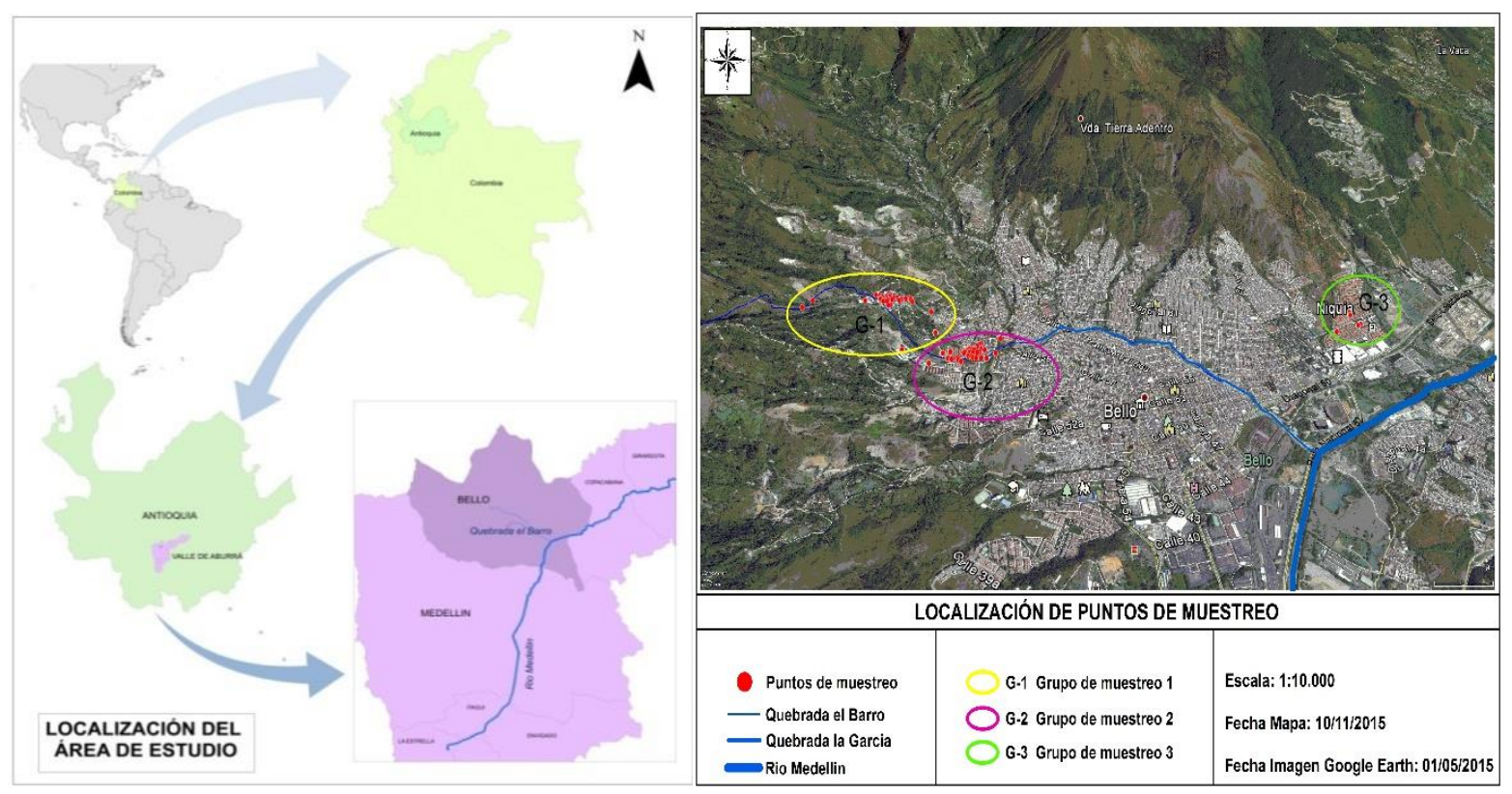

Figura 2. Localización Área de Estudio

Figura 3. Puntos de muestreo

\subsection{Participantes y procedimiento}

Se seleccionaron los participantes según dos criterios: 1) la experiencia de desastre; en este caso, la avenida torrencial ocurrida en 2005; y 2) la proximidad a una posible amenaza, representada en la quebrada El Barro con la probabilidad de que ocurra otra avenida torrencial. El estudio contó con 257 participantes adultos, 54.9\% mujeres y 45.1\% hombres; éstos, estuvieron distribuidos en tres grupos: grupo 1 (G1), población con la experiencia de desastre o emergencia (damnificados) y viven próximos a la quebrada en estudio; grupo 2, población que no tiene la experiencia de desastres y viven cerca de la quebrada (susceptibles a ser afectados); grupo 3 (G3), población sin experiencia de desastres y que no habitan en la cuenca de la quebrada, sino en otro sector del municipio (grupo control).

\subsection{Instrumento}

Se recolectó la información en campo mediante un cuestionario autoadministrado (20 minutos en promedio), conformado por preguntas de caracterización socio-demográfica, y por las siguientes escalas adaptadas a la población objeto de interés y al riesgo de avenida torrencial (avalancha): 1) apego al lugar, compuesta de 12 ítems, divididos en dos dimensiones: identidad de 
lugar y dependencia de lugar (Williams \& Vaske, 2003); 2) percepción del Riesgo, tiene como fundamento teórico el paradigma psicométrico y ha sido creada para medir la percepción del riesgo por inundación; consta de 23 ítems y 8 factores o subdimensiones: incremento global del riesgo, riesgo impredecible, temor-afectación, riesgo desconocido, renuncia a los beneficios actuales, personas expuestas, situación incontrolable y compromiso público (Terpstra, Gutteling, Geldof, \& Kappe, 2006); 3) implicación personal, permite investigar la implicación que tienen las personas frente al riesgo; se constituye de 7 ítems y consta de tres dimensiones (Lheureux, Lo Monaco, \& Guimelli, 2011); 4) estrategias de afrontamiento (coping), se usó la versión validada en México adaptada de la escala francesa "ÉchelleToulousaine de Coping" versión 1992; constituida por 26 ítems, distribuido en 2 factores: afrontamiento activo y afrontamiento pasivo (López-Vázquez \& Marván, 2004).

En cuanto a la variable de la representación social del riesgo, el instrumento contaba con una sección que tenía la palabra inductora avalancha, donde se les pidió a las personas que escribieran las palabras o expresiones que se les ocurrieran cuando pensaban en dicho fenómeno. Con esta información se realizó un análisis prototípico y categorial de la RS que se desarrollará en publicaciones posteriores.

\section{RESULTADOS}

El análisis de los datos se realizó a partir de la base de datos construida con la información recolectada en campo a través del instrumento de medición. Los principales análisis realizados fueron: 1) cálculo interno de confiabilidad del instrumento, 2) análisis de correlación de las variables, y 3) análisis de comparación de medias (ANOVA de un factor).

\subsection{Confiabilidad del Instrumento}

La tabla I presenta el alfa de Cronbach y los valores de la media para cada una de las escalas. Se observa que las escalas que mide apego de lugar (APL) y coping tienen una alta consistencia de 0.927 y 0.839 respectivamente; cuyos valores indican que estas escalas son adecuadas para investigar las variables representadas por el apego al lugar y las estrategias de afrontamiento ante una situación de riesgo. En cuanto a las escalas de percepción del riesgo (PR) y la implicación personal (IP) los resultados arrojaron una puntuación aceptable de 0.743 y 0.782; que igual permite que estas escalas sean utilizadas para la medición de las variables investigadas.

Tabla I. Propiedades psicométricas de las escalas

\begin{tabular}{lccc}
\hline Escala & Alfa de Cronbach & Número de Ítems & Media \\
\hline Apego al Lugar (APL) & .927 & 10 & 3.618 \\
\hline Percepción del Riesgo (PR) & .743 & 23 & 3.141 \\
\hline Implicación Personal (IP) & .782 & 9 & 3.294 \\
\hline Estrategias de Afrontamiento (Coping) & .839 & 26 & 3.119 \\
\hline
\end{tabular}

La estructura factorial de cada escala se verifica a través del método estadístico de análisis por componentes principales con rotación varimax ortogonal. Para validar la calidad del análisis se emplea la prueba estadística coeficiente de Kaiser-Meyer-Olkin (KMO) y la Prueba de Esfericidad de Barlett. La tabla II muestra que todos los valores son los suficientemente altos y significativos para tener la certeza que las conclusiones del análisis factorial de cada una de las escalas son satisfactorias.

Tabla II. Prueba KMO y Bartlett

\begin{tabular}{lccc}
\hline & KMO & Chi cuadrado & Sig. \\
\hline APL & .923 & 1731.61 & 0.00 \\
PR & .732 & 1248000 & 0.00 \\
IP & .818 & 486.576 & 0.00 \\
Coping & .832 & 2122000 & 0.00 \\
\hline
\end{tabular}




\subsection{Correlación entre las Variables}

En este análisis se correlacionan las siguientes variables: apego al lugar (APL), sus dimensiones identidad (APL_Ident) y dependencia (APL_Depend); percepción del riesgo (PR); implicación personal (IP), sus dimensiones valoración (IP_Valor), identificación (IP_Ident), y percepción de posibilidad de acción (IP_PPA); y las estrategias de afrontamiento (coping) con sus dimensiones coping activo y coping pasivo.

Los resultados arrojados a través de la correlación Pearson, muestran valores de alta convergencia con correlaciones positivas y significativas. Se destaca una correlación importante entre la implicación personal (IP) y coping; ésto define una relación empírica relativamente fuerte entre estas variables que corresponde a lo que conceptualmente se puede esperar. En consecuencia, se puede afirmar que el sentirse implicado está muy relacionado con el hecho de que las personas consideren que tienen las estrategias para enfrentar una determinada situación de riesgo; en este caso, las estrategias de tipo activo tiene el mayor peso y específicamente la subdimensión de la posibilidad de acción es la que aporta el mayor peso para que la variable de IP se correlacione de una manera más importante con el coping general, y particularmente con el coping activo (véase tabla III).

Tabla III. Correlación de las variables psicosociales

\begin{tabular}{|c|c|c|c|c|c|c|c|c|c|c|c|}
\hline & $\begin{array}{l}\text { APL } \\
\text { Gral }\end{array}$ & $\begin{array}{l}\text { APL } \\
\text { Ident }\end{array}$ & $\begin{array}{l}\text { APL } \\
\text { Depend }\end{array}$ & $\begin{array}{l}\text { PR } \\
\text { Gral }\end{array}$ & $\begin{array}{l}\text { IP } \\
\text { Gral }\end{array}$ & $\begin{array}{l}\text { IP } \\
\text { Ident }\end{array}$ & $\begin{array}{l}\text { IP } \\
\text { Valor }\end{array}$ & $\begin{array}{l}\text { IP } \\
\text { PPA }\end{array}$ & $\begin{array}{l}\text { Coping } \\
\text { Gral }\end{array}$ & $\begin{array}{l}\text { Coping } \\
\text { Activo }\end{array}$ & $\begin{array}{l}\text { Coping } \\
\text { Pasivo }\end{array}$ \\
\hline APL_Gral & 1 & $.938 * *$ & $.947 * *$ & $.210 * *$ & $.241 * *$ & $.209 * *$ & $.198 * *$ & $.187 * *$ & $.285^{* *}$ & $.250 * *$ & $.188 * *$ \\
\hline APL_Ident & & 1 & $.777 * *$ & $.148^{*}$ & $.216^{* *}$ & $.176^{* *}$ & $.184 * *$ & $.173 * *$ & $.239 * *$ & $.219 * *$ & $.144^{*}$ \\
\hline APL_Depend & & & 1 & $.244 * *$ & $.238 * *$ & $.217 * *$ & $.189 * *$ & $.180 * *$ & $.296 * *$ & $.251 * *$ & $.207 * *$ \\
\hline PR_Gral & & & & 1 & $.384 * *$ & $.319 * *$ & $.296^{* *}$ & $.330 * *$ & $.386^{* *}$ & $.283 * *$ & $.335 * *$ \\
\hline IP_Gral & & & & & 1 & $.864 * *$ & $.783 * *$ & $.813 * *$ & $.550 * *$ & $.573 * *$ & $.230 * *$ \\
\hline IP_Ident & & & & & & 1 & $.564 * *$ & $.541 * *$ & $.482 * *$ & $.472 * *$ & $.247 * *$ \\
\hline IP_Valor & & & & & & & 1 & $.428 * *$ & $.309 * *$ & $.335 * *$ & .111 \\
\hline NIP_PPA & & & & & & & & 1 & $.541 * *$ & $.585 * *$ & $.195 * *$ \\
\hline Coping_Gral & & & & & & & & & 1 & $.863 * *$ & $.679 * *$ \\
\hline Coping_Activo & & & & & & & & & & 1 & $.216^{* *}$ \\
\hline Coping_Pasivo & & & & & & & & & & & 1 \\
\hline
\end{tabular}

\subsection{Diferencia de medias de las variables en los grupos}

En el análisis comparativo de las medias se utilizó el análisis de varianza ANOVA de un factor; con esta prueba se contrastan las medias de los tres grupos independientes y se aplica el test post hoc de Scheffe para conocer los valores de las medias que difieren de las otras, y verificar si dicha diferencia es significativa.

Tabla IV. Comparación de medias entre los grupos

\begin{tabular}{lcccc}
\hline & \multicolumn{3}{c}{ GRUPO } \\
& G1 & G2 & G3 & Total \\
& Media & Media & Media & Media \\
\hline APL_Gral & 3,807 & 3,525 & 3,516 & 3,618 \\
APL_Ident & 3,977 & 3,734 & 3,784 & 3,833 \\
APL_Depend & 3,637 & 3,315 & 3,249 & 3,402 \\
\hline PR_Gral & 3,255 & 3,085 & 3,081 & 3,141 \\
\hline
\end{tabular}


Tabla IV. Comparación de medias entre los grupos

\begin{tabular}{lllll}
\hline IP_Gral & 3,544 & 3,131 & 3,201 & 3,294 \\
IP_Ident & 3,563 & 2,961 & 3,012 & 3,182 \\
IP_Valor & 3,770 & 3,333 & 3,514 & 3,541 \\
IP_PPA & 3,299 & 3,098 & 3,078 & 3,160 \\
\hline Coping_Gral & 3,237 & 3,047 & 3,071 & 3,119 \\
Coping_Activo & 3,426 & 3,193 & 3,347 & 3,323 \\
Coping_Pasivo & 3,017 & 2,875 & 2,749 & 2,882 \\
\hline
\end{tabular}

La tabla IV muestra la comparación global de las categorías de grupos, en donde se aprecia que el valor de la media variable APL, sobresale para G1 y difiere de las medias de G2 y G3; es evidente que estos dos últimos grupos tienen medias muy similares para la variable APL. El mismo patrón expuesto anteriormente entre los grupos, se observa para las variables PR y Coping. En cuanto la variable IP, las medias de los grupos presenta una mayor diferencia entre sí; sin embargo el grupo 1 se aleja mucho más de los grupos restantes.

Tabla V. Comparación de medias entre los grupos. ANOVA de un factor y Post Scheffe

\begin{tabular}{lccccc}
\hline Variable & $\begin{array}{c}\text { Categoría } \\
(\mathrm{I})\end{array}$ & $\begin{array}{c}\text { Categoría } \\
(\mathrm{J})\end{array}$ & $\begin{array}{c}\text { Diferencia de media } \\
(\mathrm{I}-\mathrm{J})\end{array}$ & Error std. & Sig. \\
\hline APL_Depend & $\mathrm{G} 1$ & $\mathrm{G} 2$ & .32149 & .15306 & .112 \\
& & $\mathrm{G} 3$ & $.38737^{*}$ & .15306 & .042 \\
\hline PR_Gral & $\mathrm{G} 1$ & $\mathrm{G} 2$ & .16995 & .07265 & .067 \\
& & $\mathrm{G} 3$ & .17455 & .07265 & .058 \\
\hline IP_Gral & $\mathrm{G} 1$ & $\mathrm{G} 2$ & $.41334^{*}$ & .10888 & .001 \\
& & $\mathrm{G} 3$ & $.34275^{*}$ & .10888 & .008 \\
\hline IP_Ident & G1 & G2 & $.60243^{*}$ & .13949 & .000 \\
& & $\mathrm{G} 3$ & $.55145^{*}$ & .13949 & .001 \\
\hline IP_Valor & G1 & G2 & $.43678^{*}$ & .11719 & .001 \\
& & $\mathrm{G} 3$ & .25639 & .11719 & .093 \\
\hline Coping_Gral & G1 & G2 & .19079 & .08257 & .071 \\
& & G3 & .16636 & .08257 & .133 \\
\hline Coping_Pasivo & G1 & G2 & .14175 & .09204 & .307 \\
& & G3 & $.26822^{*}$ & .09204 & .015 \\
\hline
\end{tabular}

*Nivel de significancia $\mathrm{p}<.05$

En la tabla V, se extrajeron exclusivamente los valores de la diferencia de medias que son significativos o que se encuentra muy cercano al límite de significancia; es decir los valores que representan una diferencia importante de las variables entre los grupos objetos de estudio. En este análisis, el grupo 1 (G1) presenta diferencia de medias respecto al grupo control (G3) en la subdimensión de dependencia de la variable APL, IP y coping pasivo (0.42) $(0.08)$ y $(0,015)$ respectivamente, lo que quiere decir que hay una mayor influencia de estas variables sobre G1 que en G3; ésto quiere decir que las personas expuestas y con experiencia de riesgo tienden a expresar su deseo de quedarse en su residencia (dependencia del lugar), se sienten mucho más implicados sobre el tema del riesgo, y especialmente, consideran que sus comportamientos y acciones frente a la prevención y reducción del riesgo pueden ser eficaz. Respecto al coping pasivo que hace referencia a estrategias centradas en la gestión de emociones, este grupo se diferencia con respecto al grupo 3, ya que las utiliza aún más. Asimismo, el G1 difiere del grupo 2 (G2) en la variable IP, por tanto es explicito que quienes vivieron el desastre se encuentran más implicados con la situación riesgo; sin embargo, no es evidente que los integrantes de este grupo se perciban con posibilidad de acción frente al riesgo. La variable PR se encuentra en el límite de ser significativa entre todos los grupos, así que se decide considerarla en el análisis. De esta forma, se puede afirmar que el G1 tiene una percepción del riesgo mayor que el G2 y G3, lo cual es de esperarse, ya que se deduce que la experiencia de desastre previa muy probablemente juega un rol importante en el 
incremento de la percepción del riesgo. Aunque la diferencia de medias entre G1 y G2 en relación al coping también se encuentra en el límite, igualmente se incluye en el análisis y se precisa destacar que el primer grupo expresa mayores estrategias de afrontamiento que el último.

\section{DISCUSIÓN}

Los resultados evidencian que existen correlaciones positivas y significativas entre las variables del modelo de la vulnerabilidad psicosocial: percepción del riesgo (PR), apego al lugar (APL), implicación personal (IP), y estrategias de afrontamiento (coping), lo cual va en la misma línea de estudios empíricos sobre percepción del riesgo y apego al lugar (Ruiz \& Hernández, 2014; Bonaiuto, Alves, De Dominicis, \& Petruccelli, 2016; Billig, 2006), apego al lugar y coping (Bonaiuto et al., 2016), percepción del riesgo y coping (Lopez-Vázquez \& Marvan, 2012; LópezVázquez \& Marván, 2003; López-Vásquez, 2003), implicación personal y representaciones sociales (Lopez-Vazquez \& Marvan, 2012), donde se ha demostrado la influencia directa entre estas variables, ya sea de manera positiva o negativa.

En el análisis de correlación sobresale el valor moderado-alto $(0.55)$ entre las variables implicación personal (IP) y coping; y específicamente en la subdimensión de la posibilidad de acción de la variable IP, la correlación es de 0.541; ésto se traduce en el hecho de las personas que se perciben con mayor posibilidad de acción frente a los riesgos son las que se encuentran más involucradas con los mismos (Gruev-Vintila \& Rouquette, 2007), y por tanto, es muy posible que sus acciones se vean reflejadas en las capacidades o niveles de resiliencia de la comunidad misma. En esta vía, se ha llegado a conclusiones de que la población que percibe su vulnerabilidad ante cualquier fenómeno natural es muy probable que responda a las advertencias de peligro, y en consecuencia, tome las medidas de protección pertinentes (Johnston, Lai, Houghton, \& Paton, 1999). De igual manera, algunos autores afirman que la vulnerabilidad está directamente relacionada con la percepción, a su vez con la posibilidad de reducción o amplificación, y con las acciones o respuestas respecto a ésta (Alexander, 2000); no obstante se ha discutido que la percepción del riesgo no es suficiente para explicar el grado de vulnerabilidad percibida por las personas expuestas a ciertos riesgos; ésta debe completarse con otros conceptos existentes como el de implicación personal, en el cual su rol mediador entre la percepción del riesgo y la predisposición a la acción ha sido demostrado (Navarro, Chaves, Piñeres, \& Noreña, 2016); igual ocurre con la variable apego al lugar, pues estudios muestran que ésta tiene un papel mediador y moderador entre la percepción del riesgo y el afrontamiento (Bonaiuto et al., 2016).

En este mismo sentido, el importante valor de correlación de la subdimensión precepción de acción con el coping activo (0.585), muestra que los resultados van en la misma dirección, es decir señalan la utilización de estrategias orientadas a la solución de problemas y la predisposición a comportamientos que enfrentan directamente el peligro. De hecho, se ha explicado la elección de estas estrategias a partir de la implicación que tienen las personas ante la amenaza (Navarro \& Michel-Guillou, 2014). En esta línea, estudios demuestran que en las personas expuestas a riesgos naturales, donde el nivel de estrés psicológico es alto, se predice el uso de estrategias activas (Lopez-Vazquez \& Marvan, 2012).

El análisis comparativo de las medias de las variables entre los grupos ha permitido comprobar la hipótesis respecto a la influencia que tiene la experiencia previa de desastres y la exposición ante una amenaza sobre las variables psicosociales. Los resultados indican que el grupo 1 (G1), quienes tienen la experiencia de desastres y se encuentran expuestos a los impactos de una avenida torrencial, se sienten más dependientes al lugar donde viven, se sienten más implicados, identificados, consideran más importante el riesgo, y usan mayormente estrategias de afrontamiento de tipo pasivo que el grupo 3 (G3). Esto puede indicar que efectivamente, en términos de reacción frente al riesgo, el hecho de tener una experiencia, es decir de experimentar sentimientos negativos, engendre un manejo de tipo afectivo del riesgo, lo que les permite a estas personas adaptarse psicológicamente a su situación (Terpstra, 2011). Este tipo de aspectos de la adaptación psicológica, 
emocional y cognitiva, son objeto actualmente en el contexto de la gestión de riesgos bajo el enfoque de regulación de las emociones (Gross \& Thompson, 2007). Asimismo, el G1 difiere del grupo 2 (G2), quienes están expuestos pero no tienen experiencia de desastres, en la variable IP, por tanto se comprueba que quienes vivieron el desastre se encuentran más implicados con la situación de riesgo; sin embargo, no es evidente que G1 se perciba con mayor posibilidad de acción frente al riesgo; lo cual podría explicarse en el uso de estrategias de afrontamiento, más de tipo pasivo para G1, que en este grupo sobresale significativamente en comparación con los otros dos grupos. Se resalta entonces, que las personas con experiencia de desastres usarían aquellas estrategias que les ayude a disminuir la tensión de las emociones que les genera el impacto vivido; esto tiene sentido, en la medida de que las estrategias de afrontamiento se constituye como un factor estabilizador que permite la adaptación psicosocial a los individuos, durante los periodos de estrés o situaciones de crisis (Sordes-ader, Esparbès-Pistre, \& Tap, 1997).

En relación a la percepción del riesgo (PR), los resultados muestran valores en el límite de ser significativa entre todos los grupos, por lo que se decide incluir en el análisis; de esta forma, se evidencia que el G1 tiene una percepción del riesgo mayor que G2 y G3. Estos resultados van de la línea de investigaciones sobre deslizamientos, donde se muestra que la experiencia previa y la exposición afectan la percepción de las personas e influyen en sus patrones de acciones preventivas y de afrontamiento (Landeros-Mugica, Urbina-Soria, \& Alcántara-Ayala, 2016); de igual modo, coincide con estudios sobre inundaciones que indican que la experiencia previa es el factor más influyente para elevar la percepción (Botzen, Aerts, \& van den Bergh, 2009; Tersptra, 2009) y con el planteamiento de autores, quienes señalan que la experiencia de desastre vivida afecta la percepción del peligro y la forma como lo afrontan (López-Vásquez \& Marván, 2004); en otras palabras, las experiencias previas de desastres son determinantes de la percepción y buenos predictores de las actitudes de las víctimas hacia los desastres (Siegrist \& Gutscher, 2006; Ho, Shaw, Lin, \& Chiu, 2008). Asimismo, G1 presenta mayores estrategias de afrontamiento que el G2, nuevamente coincidiendo con autores que destacan la influencia de la percepción del riesgo sobre las respuestas de afrontamiento en las personas que han vivido catástrofe (López-Vasquez, 2001). Ahora bien, un resultado que llama la atención tiene que ver con el hecho de que G1, quienes tienen la percepción del riesgo mayor, son los que se sienten más dependientes del lugar donde viven, por tanto se puede deducir que éstos serán quienes pongan mayor resistencia para dejar el lugar que habitan; ésto puede resultar paradójico y contrario al sentido común, sin embargo se puede explicar con los planteamientos de algunos autores quienes afirman que los individuos con fuertes vínculos al lugar donde viven, subestiman los efectos potenciales de una amenaza, están menos dispuestos a cambiar su lugar de residencia y muy probablemente regresan después de la ocurrencia de un desastre (Bonaiuto et al., 2016).

En síntesis, la percepción del riesgo, la implicación personal frente al mismo, y el apego al lugar donde se vive, constituyen variables que explican la tendencia de los habitantes de zonas expuestas ante una amenaza a desarrollar estrategias de afrontamiento diferentes. Estrategias que están dirigidas ya sea a la solución del problema, lo que indica una predisposición a la acción, a la adaptación efectiva (Siegrist \& Gutscher, 2008), o por el contrario más dirigidas a la gestión de las emociones, es decir una adaptación psicológica, emocional que permita manejar el estrés, sin tener que modificar nada en su entorno (Delelis, Christophe, Berjot, \& Desombre, 2011). Esto último sugiere que en términos prácticos, las autoridades que gestionan el riesgo deben propender por crear las condiciones para que las personas utilicen más estrategias activas, a través de la implicación en la solución, pues como se evidencia, sería la eficacia del comportamiento y acción percibida por las personas que podría explicar esta tendencia. Por el contrario, las personas expuestas que tienden a generar estrategias de afrontamiento pasivas tienden a desresponsabilizarse o incluso a crear sentimientos de desesperanza (Seligman, 2000), lo que los vuelve aún más vulnerables frente a la amenaza, en este caso de avenida torrencial. 


\section{CONCLUSIONES}

A partir de los resultados obtenidos se concluye que el modelo de análisis de la vulnerabilidad psicosocial propuesto es pertinente desde el punto de vista conceptual y metodológico, lo primero se refleja en la coherencia entre las variables que lo soportan, lo que apunta al hecho que no se pueden estudiar factores aislados, sino que se trata de variables que funcionan en interacción; y lo segundo se evidencia en los valores significativos de consistencia interna y validez de constructo de las escalas de medición.. Así, este modelo se sustenta a través de las correlaciones positivas y significativas entre las variables apego al lugar, percepción de riesgo, la implicación personal y estrategias de afrontamiento (coping); además, la importante correlación entre la implicación personal y el coping activo, sugiere que la implicación personal frente a los riesgos, incide significativamente en la condición de fragilidad o resiliencia de una comunidad, en tanto que las personas se sentirán con mayor capacidad para actuar en la reducción del riesgo, y sus estrategias de afrontamiento estarán esencialmente dirigidas hacia la solución de los problemas que buscarían modificar sus escenarios de riesgos. De esta manera se pone en relevancia que las variables psicosociales juegan un rol esencial en el conocimiento del riesgo y sobre la fragilidad o resiliencia de una comunidad, por tanto estas variables se constituyen en indicadores de la vulnerabilidad psicosocial

De igual manera se concluye que en la población estudiada frente a la amenaza de una avenida torrencial, la experiencia previa de desastres tiene un efecto regulador positivo sobre las variables psicosociales investigadas. En cuanto al factor exposición no es concluyente, puesto que, aunque se evidencia una influencia directa sobre G1, no sigue el mismo patrón para G2 quienes también se encuentran expuestos; y además, no se mostraron diferencias significativas con el grupo control G3 que no lo están. Finalmente, este estudio muestra la importancia de incluir la dimensión psicosocial en la gestión del riesgo de desastres, de manera que se pueda abordar la problemática del riesgo de una manera más integral, y teniendo en cuenta las condiciones y particularidades de cada comunidad, ya que éstas podrían orientar métodos y estrategias de intervención más eficaces para reducir y mitigar el riesgo en el territorio que habitan.

Además de haber confrontado empíricamente hipótesis que surgen de la literatura, éstas se formalizan a través de los resultados obtenidos, los cuales dan pistas sobre aspectos específicos que se deben trabajar en el conocimiento del riesgo, su comunicación y educación por ejemplo, orientando eventualmente medidas y estrategias de intervención en ese sentido; sin embargo, este estudio presenta límites como por ejemplo la posibilidad de comparar distintos tipos de riesgos; en efecto existen mucho factores y condiciones particulares de cada comunidades, por tanto no se puede garantizar que las respuestas a las escalas aplicadas no estén influenciadas por factores sociodemográficos o por las causas de fondo que se trabaja desde la sociología, e inclusive, por otros tipos de riesgos como la violencia o delincuencia. Este es un límite común en este tipo de estudio, ya que no es posible aislar el riesgo de todas las problemáticas de un territorio. Para trabajos próximos se sugiere ampliar la muestra o realizar un muestreo probabilístico que permita realizar generalizaciones pertinentes asociadas a las variables estudiadas; afinar los instrumentos y aplicarlos en otros tipos de fenómenos tales como: sismos, inundaciones, movimientos en masa, erosión costera, entre otros. Adicionalmente, nuevos análisis estadísticos podrían realizarse para verificar la relación causal que se propone entre variables explicativas y las variables a explicar, y además se podrían incluir otras variables como las sociodemográficas por ejemplo. Por último, se propone realizar mayores investigaciones que pongan en relevancia el rol de la naturaleza del fenómeno frente a las variables psicosociales, y especialmente en relación a los fenómenos que se encuentran en aumento debido a los cambios ambientales globales. 


\section{REFERENCIAS}

Abric, J. (1994). Pratiques sociales et représentations. Paris: Presses universitaires de France.

Bernardo, F. (2013). Impact of place attachment on risk perception: Exploring the multidimensionality of risk and its magnitude. Estudios de Psicología: Studies in Psychology, 34(3), 323-329.

Bickerstaff, K. (2004). Risk perception research: socio-cultural perspectives on the public experience of air pollution. Environment International, 30(6), 827-40.

Billig, M. (2006). Is My Home My Castle? Place Attachment, Risk Perception, and Religious Faith. Environment and Behavior, 38(November), 248.

Birkmann, J. (2006). Measuring vulnerability to promote disaster-resilient societies : Conceptual frameworks and definitions. In J. Birkmann (Ed.), Measuring Vulnerability to Natural Hazards: Towards Disaster Resilient Societies (pp. 9-54). Tokyo - New york - Paris: United Nations University Press.

Bonaiuto, M., Alves, S., De Dominicis, S., \& Petruccelli, I. (2016). Place attachment and natural environmental risk: Research review and agenda. Journal of Environmental Psychology, 48, 33-53.

Botzen, W. J. W., Aerts, J. C. J. H., \& van den Bergh, J. C. J. M. (2009). Dependence of flood risk perceptions on socioeconomic and objective risk factors. Water Resources Research, 45 W10440.

Brown, B., \& Perkins, D. (1992). Disruptions in Place Attachment. In I. Altman \& S. M. Low (Eds.), Place Attachment (pp. 279-304). Boston, MA: Springer US.

Cardona, O. (2001). La necesidad de repensar de manera holística los conceptos de vulnerabilidad y riesgo. Una crítica y una revisión necesaria para la gestión. Centro de Estudios Sobre Desastres Y Riesgos, 1-18.

Cardona, O. D., Bertoni, J. C., Gibbs, T., Hermelin, M., \& Lavell, A. (2010). Entendimiento y Gestión del Riesgo Asociado a las Amenazas Naturales: Un Enfoque Científico Integral para América Latina y el Caribe.

Catalán-Vázquez, M., Ms, M., Riojas-Rodríguez, H., C, M., Jarillo-Soto, E. C., \& DelgadilloGutiérrez, H. J. (2009). Percepción de riesgo a la salud por contaminación del aire en adolescentes de la Ciudad de México. Salud Pública de México, 51(2), 148-156.

Creswell, J. (2002). Research design qualitative, quantitative, and mixed methods approaches. Thousands Oaks: Sage Publications.

Delelis, G., Christophe, V., Berjot, S., \& Desombre, C. (2011). Stratégies de régulation émotionnelle et de coping : quels liens ? Bulletin de Psychologie, Numéro 515(5), 471.

Douglas, M., \& Wildavsky, A. B. (1982). Risk and culture : an essay on the selection of technical and environmental dangers. Berkeley: University of California Press.

Feuerstein, M., Labbé, E. E., \& Kuczmierczyk, A. (1986). Health psychology: A psychobiological perspective. New York: Plenum Press.

Fischhoff, B., Slovic, P., Lichtenstein, S., Read, S., \& Combs, B. (1978). How safe is safe enough? A psychometric study of attitudes towards technological risks and benefits. Policy Sciences, $9(2)$, $127-152$. 
Gruev-Vintila, A., \& Rouquette, M.-L. (2007). Social Thinking about Collective Risk: How Do Risk-related Practice and Personal Involvement Impact Its Social Representations? Journal of Risk Research, 10(4), 555-581.

Hidalgo, M., \& Hernández, B. (2001). Place Attachment: Conceptual and Empirical Questions. Journal of Environmental Psychology, 21(3), 273-281.

Ho, M., Shaw, D., Lin, S., \& Chiu, Y. (2008). How do disaster characteristics influence risk perception? Risk Analysis : An Official Publication of the Society for Risk Analysis, 28(3), 635-43.

Jodelet, D. (1986). La representación social: fenómenos, concepto y teoría. (S. Moscovici, Ed.), Psicología social II. Barcelona.

Keipi, K., Mora, S., \& Bastidas, P. (2005). Gestión de riesgo derivado de amenazas naturales en proyectos de desarrollo: lista de preguntas de verificación., 55.

Kellens, W., Zaalberg, R., Neutens, T., Vanneuville, W., \& De Maeyer, P. (2011). An analysis of the public perception of flood risk on the Belgian coast. Risk Analysis : An Official Publication of the Society for Risk Analysis, 31(7), 1055-68.

Landeros-Mugica, K., Urbina-Soria, J., \& Alcántara-Ayala, I. (2016). The good, the bad and the ugly: on the interactions among experience, exposure and commitment with reference to landslide risk perception in México. Natural Hazards, 80(3), 1515-1537.

Lazarus, R. (1999). Stress and emotion : a new synthesis. New York: Springer Pub. Co.

Lazarus, R., \& Folkman, S. (1984). Stress, appraisal, and coping. New York: Springer Pub. Co.

Lazarus, R., \& Folkman, S. (1986). Estrés y procesos cognitivos. Barcelona: Martínez Roca.

Lerner, J. S., Gonzalez, R. M., Small, D. a., \& Fischhoff, B. (2003). Effects of fear and anger on perceived risks of terrorism: A national field experiment. Psychological Science, 14(2), 144-150.

Lewicka, M. (2011). Place attachment: How far have we come in the last 40 years? Journal of Environmental Psychology, 31(3), 207-230.

Lheureux, F., Lo Monaco, G., \& Guimelli, C. (2011). Entre Représentations Sociales et Intentions de Pratiques: L'Implication. Interamerican Journal of Psychology, 45(1994), 61-76.

Lindell, M. K., Zhang, Y., \& Hwang, S. N. (2009). Hazard Proximity or Risk Perception? Evaluating Effects of Natural and Technological Hazards on Housing Values. Environment and Behavior, 42(5), 597-624.

Lindsay, P. H., \& Norman, D. A. (1980). Traitement de l'information et comportement humain: une introduction à la psychologie. Ed. Etudes vivantes.

López-Vasquez, E. (2001). Risk perception interactions in stress and coping facing extreme risks. Environmental Management and Health, 12(2), 122-133.

Lopez-Vazquez, E. (2009). Risk perception and coping strategies for risk from Popocatepetl Volcano, Mexico. Geofisica Internacional, 48(1), 133-147.

Lopez-Vazquez, E., Marvan, L., Flores, F., \& Peyrefitte, A. (2008). Volcanic Risk Exposure, Feelings of Insecurity, Stress, and Coping Strategies in Mexico. Journal of Applied Social Psychology, 38(12).

Lopez-Vazquez, E., \& Marvan, M. L. (2012). Volcanic Risk Perception, Locus of Control, Stress and Coping Responses of People Living Near the Popocatepetl Volcano in Mexico. Journal of Risk Analysis and Crisis Response, 2(1), 3.

López-Vázquez, E., \& Marván, M. L. (2003). Risk perception, stress y coping strategies in two catastrophe risk situations. Social Behavior and Personality, 31(1), 61-70. 
López-Vázquez, E., \& Marván, M. L. (2004). Validación de una escala de afrontamiento frente a riesgos extremos. Salud Pública de México, 46(3), 216-221.

Moos, R. H., \& Billings, A. G. (1982). Conceptualizing and measuring coping resource and processes. In L. Goldberger (Ed.), Handbook of stress : theoretical and clinical aspects. New York ;London: Free Press; Collier Macmillan.

Mora, M. (2002). La teoría de las representaciones sociales de Serge Moscovici. Athenea Digital, 2(1979), 1-25.

Narváez, L., Lavell, A., \& Pérez, G. (2009). La Gestión del Riesgo de Desastres: Un enfoque basado en procesos. Comunidad Andina. Secretaría General; Proyecto Apoyo a la Prevención de Desastres en la Comunidad Andina (PREDECAN).

Navarro, O. (2004). Representación Social del Agua y de sus Usos. Psicología Desde El Caribe. Universidad Del Norte, (14), 222-236.

Navarro, O., Chaves, L., Piñeres, J. D., \& Noreña, M. I. (2016). Risk perception and coping strategies in population exposed and not exposed to flooding risk. Revista Interamericana de Psicologia/Interamerican Journal of Psychology, 50(3), 331-346.

Puy, A., \& Aragonés, J. (1997). Percepción social de los riesgos y gestión de las emergencias ambientales. Revista Desastre y Sociedad, (5), 5-24.

Ruiz, C., \& Hernández, B. (2014). Emotions and coping strategies during an episode of volcanic activity and their relations to place attachment. Journal of Environmental Psychology, 38, 279-287.

Siegrist, M., \& Gutscher, H. (2006). Flooding risks: a comparison of lay people's perceptions and expert's assessments in Switzerland. Risk Analysis : An Official Publication of the Society for Risk Analysis, 26(4), 971-9.

Siegrist, M., \& Gutscher, H. (2008). Natural Hazards and Motivation for Mitigation Behavior: People Cannot Predict the Affect Evoked by a Severe Flood. Risk Analysis, 28(3), 771-778.

Sjöberg, L. (2002). Are received risk perception models alive and well? Risk Analysis, 22(4), 665669.

Slovic, P. (1987). Perception of risk. Science. Vol. 236 No. 4799, 1987, pp. 280-285

Slovic, P., Fischhoff, B., \& Lichtenstein, S. (1980). Facts and fears: Understanding perceived risk. In Societal risk assessment: How Safe is Safe Enough? (R. C. Schwing \& W. A. Albers, Eds.). Boston, MA: Springer US.

Slovic, P., \& Peters, E. (2006). Risk perception and affect. Current Directions in Psychological Science, 15(6), 322-325.

Sordes-ader, F., Esparbès-Pistre, S., \& Tap, P. (1997). Adaptation et stratégies de coping à l'adolescence. Etude différentielle selon le sexe et l'âge. Spirale - Revue de Recherches En Education, 20, 131-153.

Terpstra, T. (2009). Flood Preparedness. Thoughts, feelings and intentions of the Dutch public.

Terpstra, T. (2011). Emotions, Trust, and Perceived Risk: Affective and Cognitive Routes to Flood Preparedness Behavior. Risk Analysis, 31(10), 1658-1675.

Terpstra, T., Gutteling, J. M., Geldof, G. D., \& Kappe, L. J. (2006). The perception of flood risk and water nuisance. Water Science and Technology, 54(August), 431-439.

Unidas, N. (2013). Informe de Evaluacion Global sobre la Reduccion del Riesgo de Desastres. United Nations Pubns. 
Wandersman, A. H., \& Hallman, W. K. (1993). Are people acting irrationally? Understanding public concerns about environmental threats. American Psychologist, 48(6), 681-686.

Williams, D., \& Vaske, J. (2003). The Measurement of Place Attachment: Validity and Generalizability of a Psychometric Approach. Forest Science, 49(6), 830-840.

Yamin, L. E., Ghesquiere, F., Cardona, O. D., Ordaz, M. G., \& Mundial, B. (2013). Modelación probabilista para la gestión del riesgo de desastre. El caso de Bogotá, Colombia. 
Data de aceite: 02/08/2017 